\title{
Fallo terapéutico del bloqueo de ramo medial lumbar y su relación con la obesidad. Estudio de cohorte retrospectivo
}

\section{Therapeutic failure of the lumbar medial branch block and its relation to obesity. Retrospective cohort study}

\author{
M. E. Bravo Peña ${ }^{1}$, J. H. Arias Botero², J. Buriticá Franco ${ }^{3}$, C. Salazar Galeano ${ }^{3}$ y A. F. Yépes Ríos ${ }^{4}$
}

${ }^{1}$ Programa de Especialización en Dolor y Cuidados Paliativos. Universidad CES. Medellín, Colombia.

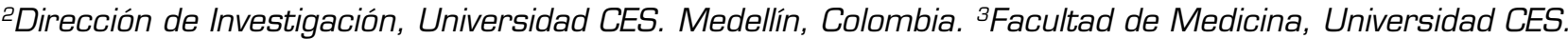
Medellín, Colombia. ${ }^{4}$ Instituto Colombiano del Dolor INCODOL. Medellín, Colombia

\section{ABSTRACT}

Objective: To compare the frequency of therapeutic failure of bilateral lumbar facet block in patients with chronic axial low back pain, obese with non-obese, in a chronic pain care center in western Colombia for one year.

Materials and methods: An observational analytical follow-up study of a retrospective cohort was performed. electronic medical records of patients were reviewed, the patients were over 18 years old, of both sexes, bilateral lumbar facet block was performed due to chronic axial low back pain, they had a record of body mass index, and post-procedure control between the 1 st of January and December 31, 2018.

Pain score values were recorded by the Visual Analog Scale (VAS) before and after the procedure. Therapeutic failure was considered when the improvement in pain was less than $50 \%$ of the initial VAS value (4), and an analysis was applied for other modifying variables reported in the literature.

Results: 190 patients were included, with an average age of 56.8 years ( \pm 13.5 ), $49.5 \%$ female, $66.3 \%$ were employees, $17.4 \%$ housewives, $8,9 \%$ independent, and $7,4 \%$ pensioners.

$29 \%$ showed a normal BMI, $42.6 \%$ overweight, and $28.4 \%$ obesity. The most frequent monotherapy was acetaminophen in $12.1 \%$, a third of the patients had received physical therapy. $23.7 \%$ had a history of previous interventionism and most of these had a positive response.

\section{RESUMEN}

Objetivo: Comparar la frecuencia de fallo terapéutico del bloqueo de ramo medial lumbar bilateral en pacientes con dolor lumbar crónico axial, obesos con no obesos, en un centro de atención de dolor crónico del occidente colombiano durante un año.

Materiales y métodos: Se realizó un estudio analítico observacional de seguimiento a una cohorte retrospectiva. Se revisaron las historias clínicas electrónicas de pacientes, mayores de 18 años, de ambos sexos, a quienes se les realizó bloqueo de ramo medial lumbar bilateral por dolor lumbar crónico axial, que contaron con registro de índice de masa corporal, y control posprocedimiento entre el 1 de enero y el 31 de diciembre de 2018.

Se registraron los valores de calificación de dolor por la Escala Visual Análoga (EVA) antes y después del procedimiento. Se consideró fallo terapéutico cuando la mejoría de dolor fue menor al $50 \%$ del valor de EVA inicial (4), y se aplicó un análisis por otras variables modificadoras reportadas en la literatura.

Resultados: Se incluyeron 190 pacientes, con una edad promedio de 56,8 años $( \pm 13,5)$, el $49,5 \%$ de sexo femenino, el $66,3 \%$ fueron empleados, el 17,4\% amas de casa, el 8,9\% independientes, y el 7,4\% pensionados.

El 29 \% mostró un IMC normal, el 42,6 \% sobrepeso y el $28,4 \%$ obesidad. La monoterapia más frecuente fue el acetaminofén en un 12,1\%, una tercera parte

Recibido: 16-06-2020

Aceptado: 07-09-2020

Correspondencia: Mary Elizabeth Bravo Peña maryelizabethbravopeña@gmail.com
Bravo Peña ME, Arias Botero JH, Buriticá Franco J, Salazar Galeano C, Yépes Ríos AF. Fallo terapéutico del bloqueo de ramo medial lumbar y su relación con la obesidad. Estudio de cohorte retrospectivo. Rev Soc Esp Dolor. 2020;27(5):298-305 
The percentage of therapeutic failure was $54.7 \%$ (104 patients); and the variables that showed statistical association with this were: age $(p=0.014)$, the previous response to interventionism ( $p=0.014)$, and time of evolution of pain ( $p=0.045$ ).

The percentage of obesity among those who presented therapeutic failure was $76.9 \%$, while in those who did not present it, it was $64 \%$ [ $=0.05$ ).

In the multivariate analysis, the variables that showed statistical significance against therapeutic failure were: age younger than 50 years $\mathrm{OR} 2.7, \mathrm{Cl}[1.37-5.34]$ $p=0.004$; and overweight OR 2.36, IC [1.11 - 5.04], $\mathrm{p}=0.026$.

Conclusion: In this study, evidence that being overweight is associated with therapeutic failure in lumbar facet block was found, although the same did not happen with obesity.

Key words: Obesity, low back pain, medial branch block, zygapophyseal joint, spine. de los pacientes había recibido terapia física. El 23,7\% tuvo historia de intervencionismo previa y la mayoría de estos habian tenido una respuesta positiva.

El porcentaje de fallo terapéutico fue de 54,7\% (104 pacientes), y las variables que mostraron asociación estadística con esta fueron: la edad ( $p=0,014)$, la respuesta previa al intervencionismo $(p=0,014)$ y tiempo de evolución del dolor $(p=0,045)$.

El porcentaje de obesidad entre los que presentaron fallo terapéutico fue del 76,9\%, mientras que en los que no la presentaron fue del $64 \%$ ( $p=0,05)$.

En el análisis multivariado, las variables que mostraron significancia estadística frente a fallo terapéutico fueron: edad menor de 50 años OR 2,7, IC [1,37 - 5,34] $p=0,004$, y sobrepeso OR 2,36, IC [1,11 - 5,04], $\mathrm{p}=0,026$.

Conclusión: En este estudio se encontró evidencia de que el sobrepeso se asocia al fallo terapéutico en el bloqueo de ramo medial lumbar, aunque no sucedió lo mismo con la obesidad.

Palabras clave: Obesidad, dolor lumbar, bloqueo rama medial, articulación cigapofisaria, columna.

\section{INTRODUCCIÓN}

En el mundo el dolor lumbar crónico alcanza una prevalencia del $84 \%$, y constituye una gran carga económica y una causa de discapacidad considerable (1). Esta patología ha evidenciado una fuerte asociación con la obesidad, la cual también representa un grave problema de salud pública [2].

Las articulaciones facetarias han sido descritas como una causa de dolor mecánico lumbar, crónico, axial, que varía entre el 15 y el $45 \%$ [3]. Los bloqueos de ramo medial son el segundo tipo de procedimiento más frecuente en el tratamiento del dolor en los Estados Unidos, siendo el único método confiable para identificar las articulaciones facetarias como fuente de dolor [4].

Existe poca evidencia acerca de la frecuencia de fallo terapéutico del bloqueo de ramo medial en el dolor de origen facetario, pero muchos factores pueden influir en la perpetuación del dolor,y el mejor método para comprobar el origen es el propio bloqueo.

A nivel global, la evidencia es controversial en cuanto al impacto de la obesidad en los procedimientos intervencionistas en dolor [2]. Ospina y cols. han mostrado que el bloqueo de ramo medial no exitoso en dolor lumbar axial crónico presuntamente por clínica de origen facetario se presenta aproximadamente en un $30 \%$ de pacientes, sin embargo las variables que influyen en el fallo terapéutico se han estudiado poco en nuestro medio [5].

Diferentes series han mostrado que la obesidad es un factor que influye en el dolor lumbar persistente (3); pero se desconoce si tiene un papel determinante en el fallo terapéutico del intervencionismo en dolor lumbar crónico axial.
El objetivo del presente estudio es describir la frecuencia de fallo terapéutico del bloqueo de ramo medial lumbar bilateral por dolor lumbar crónico axial en un centro de atención de dolor crónico del occidente colombiano, comparándola entre pacientes obesos y no obesos.

\section{MATERIALES Y MÉTODOS}

Se realizó un estudio retrospectivo de seguimiento a una cohorte de pacientes con dolor lumbar crónico axial a quienes se les realizó bloqueo de ramo medial como parte de su tratamiento. A partir del listado de procedimientos de la institución, se identificaron los pacientes sometidos a bloqueo de ramo medial lumbar bilateral entre el 1 de enero y el 31 de diciembre de 2018. Se excluyeron pacientes menores a 18 años y a quienes en la historia clínica no se contaba con información del seguimiento posterior al procedimiento.

La institución cuenta con un protocolo de bloqueo de ramo medial lumbar guiado por fluoroscopia en el cual, con una aguja raquídea calibre $22 \mathrm{G}$, se ubica anatómicamente el ramo medial bilateral en 13-14 y dorsal de 15, se aplica una mezcla de anestésico local (bupivacaína al 0,5\%) más un esteroide de depósito [acetato de metilprednisolona) en un volumen menor a 1 cc.

Se revisaron las historias clínicas electrónicas de los pacientes que cumplieron los criterios de inclusión. La información fue recolectada por médicos que cursan la subespecialidad de dolor y por estudiantes de medicina, quienes fueron previamente entrenados y estandarizados en el formulario de recolección. Se recogió información de la edad, sexo, ocupación, variables antropométricas, comorbilidades asocia- 
das, tiempo de evolución del dolor, medicamentos utilizados, terapia física e intervencionismo previos. El indice de masa corporal (IMC) se categorizó así: normal menor a 25, sobrepeso entre 25 y 30 y obesidad mayor a $30 \mathrm{~kg} / \mathrm{m}^{2}$. Además, se buscó anotaciones en la historia clínica asociadas a reclamación laboral secundaria al dolor lumbar para analizar un posible efecto confusor.

Se registraron los valores de calificación de dolor por la Escala Visual Análoga [EVA] antes y después del procedimiento. Se consideró fallo terapéutico cuando la mejoría de dolor fue menor a $50 \%$ del valor de EVA antes del procedimiento (4).

El protocolo del estudio fue aprobado por el Comité de Investigación e Innovación de la universidad CES (acta . $^{\circ}$ 223) y por la institución de manejo de dolor donde se desarrolló la investigación.

\section{Análisis estadístico}

Para describir las características de la población se reportaron promedio y desviación estándar, para las variables cuantitativas y frecuencias absolutas y relativas para las variables cualitativas. Para comparar las variables estudiadas entre los grupos (fallo terapéutico vs. no falla), se utilizó la prueba de Chi cuadrado. Se consideró significativo un valor de $p \leq 0,05$. Con el fin de estimar la asociación entre obesidad y el fallo terapéutico, se realizó un modelo de regresión logística binaria ajustando por las variables del paciente, características del dolor y uso de medicamentos. Se reportan los RR ajustados con su intervalo de confianza del $95 \%$. Los análisis se realizaron en SPSS v21.

\section{RESULTADOS}

En el periodo de estudio 190 pacientes cumplieron los criterios para ingresar al estudio. La edad promedio fue de 56,8 años con una desviación estándar de 13,5 años; el 50,5 \% (96 pacientes) eran hombres. La mayoría $(66,3 \%$ ) eran empleados. Solamente el 4,2 \% tenía una reclamación laboral relacionada con el dolor lumbar. El $50 \%$ de los pacientes presentaban un dolor de un año o menos de evolución. Los tratamientos farmacológicos recibidos fueron variados, siendo más frecuentes las combinaciones de medicamentos. La monoterapia más frecuentemente fue el acetaminofén $(12,1 \%)$. Solamente una tercera parte de los pacientes habían recibido terapia física para el tratamiento del dolor lumbar. El 23,7\% tuvieron historia de intervencionismo previa y, de estos, el $73 \%$ habian tenido una respuesta positiva a este manejo (Tabla I). La frecuencia de fallo terapéutico fue del 54,7\% (104 pacientes).

En el análisis bivariado (Tabla II) presentaron asociación estadísticamente significativa con el fallo terapéutico la edad menor a 50 años ( $p=0,014)$, la respuesta previa al intervencionismo $(p=0,014)$ y tiempo de evolución del dolor ( $p=0,045)$. La obesidad (IMC > 30) no se asoció a fallo terapéutico, aunque la frecuencia de fallo de bloqueo fue mayor entre los pacientes con sobrepeso que entre los de peso normal $[61,7$ vs. 43,6 \%) (Tabla II). Cuando se agrupan las categorías, el $77 \%$ de quienes presentaron fallo terapéutico tenían sobrepeso u obesidad, mientras estas dos estaban presentes en el $64 \%$ de quienes el bloqueo fue efectivo $(p=0,05)$.

En el modelo multivariado, además de la clasificación del IMC, se ingresaron las covariables que se consideraron podrían asociarse a fallo terapéutico incluyendo edad, sexo, tiempo de evolución, terapia física, antecedente de intervencionismo. Inicialmente se ingresaron inicialmente las combinaciones "acetaminofén-opioide" y "acetaminofén-AINE" pero se retiraron por no ser significativas en el multivariado, además de no aportar varianza adicional al modelo. Se evidenció que las variables que mostraron asociación con el fallo terapéutico fueron la edad menor de 50 años (RR 1,48 IC $95 \%$ $[1,16-1,72] p=0,004) ; y$ tener sobrepeso (RR 1,48 IC $95 \%[1,06-1,83], p=0,026$ ] (Tabla III).

Un análisis adicional para el subgrupo de pacientes que tuvieron bloqueos previos evidenció relación de la fallo terapéutico con no haber recibido terapia física [OR $7,33$ IC $95 \%[1,15-46,55]]$ y con la falta de mejoría a un bloqueo anterior [OR 14,27 IC 95 \% [1,93-105,4].

\section{DISCUSIÓN}

El presente estudio reporta una frecuencia de fallo terapéutico del bloqueo de ramo medial mayor a la de otros reportes. Ospina y cols. reportan una mejoría en el $78 \%$ de los pacientes, sin embargo, el método para evaluación de mejoría fue cualitativo, lo que no permite comparar los resultados (5). Consideramos que utilizar un umbral de mejoría mayor a $50 \%$ del dolor basal es una medida más adecuada de efectividad, teniendo en cuenta la posibilidad de efecto placebo [4], además del carácter terapéutico y diagnóstico del procedimiento [6].

Hay que anotar que el porcentaje de falla puede estar afectado por diferentes variables como edad, género, etc., variando entre poblaciones [7]. También se ha analizado la variación del porcentaje de fallo terapéutico relacionada con la técnica; por ejemplo intrarticular, de rama medial, con o sin esteroide, ecoguiado, etc. $[8,10]$. En este estudio se tomó una técnica estandarizada, bilateral y de ramo medial para hacer el grupo de estudio más homogéneo. Por otro lado, es importante mencionar que en la evaluación del resultado no se midió el tiempo de respuesta dado el carácter retrospectivo del estudio, sin embargo, los periodos de seguimiento oscilaron entre uno y tres meses.

El fallo terapéutico se presentó en un porcentaje mayor en los individuos con sobrepeso, pero no en aquellos con obesidad, acorde con lo reportado por Cohen y cols. (11), quienes reportan que un IMC > 30 no fue un factor relacionado con el éxito de la radiofrecuencia facetaria lumbar.

Existen diferentes reportes acerca de la relación del IMC anormal con el dolor lumbar en diferentes aspectos: Manchikanti menciona que las articulaciones facetarias son una causa importante de dolor lumbar persistente indistintamente en pacientes obesos y con IMC normal [3]; Ibrahimi, en 2015, reporta que la obesidad no tiene influencia directa en el dolor lumbar bajo de espalda, pero podría prolongar el tiempo de recuperación (12). 
TABLA I

CARACTERÍSTICAS DEMOGRÁFICAS Y CLÍNICAS DE LOS PACIENTES

\begin{tabular}{|c|c|c|}
\hline Edad Prom $[ \pm$ DS ] & 56,8 & {$[ \pm 13,5]$} \\
\hline \multicolumn{3}{|l|}{ Sexo n (\%) } \\
\hline Masculino & 96 & {$[50,5]$} \\
\hline Femenino & 94 & {$[49,5]$} \\
\hline \multicolumn{3}{|l|}{ Ocupación n [\%] } \\
\hline Empleado & 126 & {$[66,3)$} \\
\hline Ama de casa & 33 & {$[17,4]$} \\
\hline Independiente & 17 & {$[8,9]$} \\
\hline Pensionado & 14 & {$[7,4)$} \\
\hline \multicolumn{3}{|l|}{ Conflicto laboral n [\%] } \\
\hline No & 182 & $(95,8)$ \\
\hline Sí & 8 & {$[4,2]$} \\
\hline \multicolumn{3}{|l|}{ Fumador n (\%) } \\
\hline No & 179 & $(94,2)$ \\
\hline Sí & 11 & {$[5,8]$} \\
\hline \multicolumn{3}{|l|}{ IMC n [\%] } \\
\hline Normal & 55 & (29) \\
\hline Sobrepeso & 81 & {$[42,6]$} \\
\hline Obesidad & 54 & $(28,4)$ \\
\hline Tiempo de evolución del dolor Me (Riq) & 1 & $(1-5)$ \\
\hline \multicolumn{3}{|l|}{ Tipo de enfermedad asociada n (\%) } \\
\hline No musculoesqueléticas & 108 & $(56,9)$ \\
\hline Musculoesqueléticas & 19 & (10) \\
\hline Ninguna & 63 & $(33,1)$ \\
\hline \multicolumn{3}{|l|}{ Medicamentos n [\%] } \\
\hline Acetaminofén & 23 & $(12,1)$ \\
\hline AINE & 10 & {$[5,3]$} \\
\hline Opioide & 3 & $(1,6)$ \\
\hline Antineuropático & 4 & {$[2,1]$} \\
\hline Acetaminofén/Opioide & 40 & {$[21,1]$} \\
\hline AINE/Opioide & 1 & {$[0,5]$} \\
\hline Opioide/Antineuropático & 4 & {$[2,1]$} \\
\hline Acetaminofén/AINE & 13 & $(6,8)$ \\
\hline Acetaminofén/Antineuropático & 6 & {$[3,2]$} \\
\hline Otros & 86 & {$[45,3]$} \\
\hline \multicolumn{3}{|l|}{ Terapia física n [\%] } \\
\hline Sí & 60 & {$[31,6]$} \\
\hline No & 130 & $(68,4)$ \\
\hline \multicolumn{3}{|l|}{ Historia de intervencionismo $\mathrm{n}[\%]$} \\
\hline Sí & 45 & $(23,7)$ \\
\hline No & 145 & $(76,3)$ \\
\hline \multicolumn{3}{|c|}{ Respuesta al intervencionismo previo * $\mathrm{n}$ (\%) } \\
\hline Sí & 33 & $(73,3)$ \\
\hline No & 12 & $(26,7)$ \\
\hline
\end{tabular}

*Calculado para el total que recibieron intervencionismo anterior.

Prom: promedio. DS: desviación estándar. Riq: rango intercuartílico. IMC: índice de masa corporal. 
TABLA II

CARACTERÍSTICAS DE LOS PACIENTES SEGÚN FALLO TERAPÉUTICO

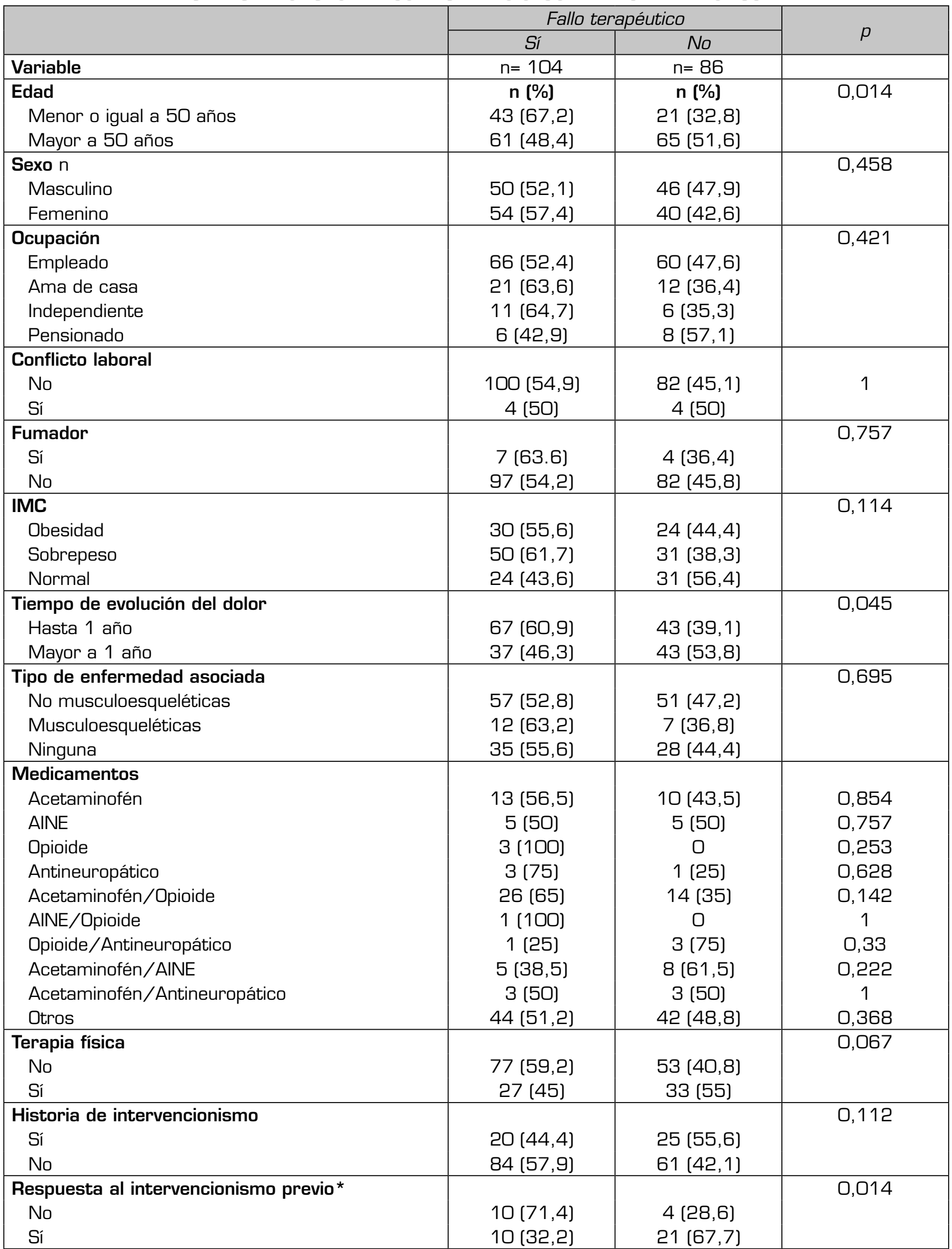


TABLA III

FACTORES ASOCIADOS CON EL FALLO TERAPÉUTICO DEL BLOQUEO DEL RAMO MEDIAL

\begin{tabular}{|c|c|c|c|}
\hline Covariables & $R R$ & IC $95 \%$ & $p$ \\
\hline \multicolumn{4}{|l|}{ Edad } \\
\hline Menor a 50 años & 1,48 & {$[1,16-1,72]$} & 0,004 \\
\hline Mayor a 50 años & 1 & & \\
\hline \multicolumn{4}{|l|}{ Sexo } \\
\hline Masculino & 0,81 & {$[0,55-1,08]$} & 0,17 \\
\hline Femenino & 1 & & \\
\hline \multicolumn{4}{|l|}{ Tiempo de evolución } \\
\hline Hasta 1 año & 1,28 & {$[0,95-1,58]$} & 0,095 \\
\hline Mas de 1 año & 1 & & \\
\hline \multicolumn{4}{|l|}{ Terapia física } \\
\hline No & 1,32 & {$[0,95-1,65]$} & 0,09 \\
\hline Sí & 1 & & \\
\hline \multicolumn{4}{|l|}{ Historia de intervencionismo } \\
\hline Sí & 0,82 & {$[0,53-1,13]$} & 0,264 \\
\hline No & 1 & & \\
\hline \multicolumn{4}{|l|}{ Categorías IMC } \\
\hline Obesidad & 1,21 & {$[0,77-1,64]$} & 0,366 \\
\hline Sobrepeso & 1,48 & {$[1,06-1,83]$} & 0,026 \\
\hline Normal & 1 & & \\
\hline
\end{tabular}

Test de bondad de ajuste (Hosmer-Lemeshow) $p=0,566$.

Adicionalmente Shiri y cols. (13) determinan factores de riesgo para el dolor lumbar, describiendo una correlación con el índice de masa corporal.

Debido a los estudios que relacionan dolor lumbar y obesidad, hay una tendencia fuerte a creer que la obesidad pudiera ser un factor de mal pronóstico para el éxito terapéutico de los procedimientos intervencionistas. Sin tener evidencia clara y contundente, esto podría llevar a una restricción de los bloqueos en los pacientes obesos, sin embargo es necesario tener en cuenta que otros factores, como el diagnóstico clínico adecuado, también influyen directamente en el éxito del bloqueo de ramo medial en los pacientes obesos (11).

Asimismo, el fallo terapéutico fue menos frecuente en los pacientes que realizaron terapia física previa al procedimiento, particularmente para el subgrupo que había recibido intervencionismo previo; esto apoya lo reportado en la literatura por o Gomes-Neto y cols. (14) en un metanálisis que demuestra que la terapia física es útil en el tratamiento del dolor lumbar axial (15), y que el manejo multidisciplinario trae mejores resultados en el grupo de pacientes (4) con dolor bajo de espalda, sometidos a intervencionismo como alternativa de manejo del dolor (16).

También se evidenció que una buena respuesta previa al intervencionismo y un mayor tiempo de evolución del dolor se asociaron de manera estadísticamente significativa a un menor fallo terapéutico, probable- mente debido a que el punto de corte que se tomó fue de un año, y la etiología del dolor menor a este tiempo pudo ser con menor probabiliadad de origen facetario. Se ha mencionado la relación entre el tiempo de evolución y el pronóstico del dolor de manera similar en un estudio en facetas cervicales realizado por Cohen y cols. en 2007 (11), aunque de manera inversa el mayor tiempo de evolución se relacionó con un mal pronóstico.

En este estudio, los mayores de 50 años mostraron un mayor porcentaje de éxito del procedimiento que los menores de 50 años. Esto es coherente con lo reportado en la literatura, aunque con diferentes puntos de corte para la edad. Este estudio tomó como valor de referencia la revision de Manchikanti [7]. Estos resultados son explicados probablemente porque en el grupo de mayor edad la etiología del dolor lumbar es principalmente de origen facetario $(12,17)$.

Para las personas que tomaban monoterapia no hubo diferencia en el fallo terapéutico. Las variables de tratamiento con medicamentos no se ingresaron al modelo por no ser significativas en el bivariado, contrariamente a lo mencionado en estudios previos [8], donde el manejo farmacológico se ha reportado como una variable modificadora.

Además, se midieron variables como el género, se encontró igual proporción para masculino y femenino en contraposición a lo reportado en otros estudios, como el de Manchicanti, donde se evidencia mayor prevalen- 
cia de dolor lumbar en las mujeres (3). La edad promedio de los pacientes fue alrededor de los 50 años, lo que se ha mencionado de manera similar en otros estudios (18).

Mas de la mitad de los pacientes del estudio estaban activos laboralmente; siendo principalmente empleados, la mayoría no se presentó conflicto laboral, aspecto conocido por influir probablemente en el desenlace del bloqueo [8]. En este estudio no se encontraron muchos pacientes con conflicto laboral, lo que podría explicarse porque al ser un conocido factor de mal pronóstico se evita realizar el procedimiento de manera frecuente.

Una fortaleza importante del estudio es el alto número de pacientes con que cuenta la institución, dedicada exclusivamente al manejo de pacientes con dolor crónico; esto permitió excluir los bloqueos combinados, los bloqueos unilaterales y dejar los bloqueos de ramo medial lumbares bilaterales puros estandarizados, lo que hace el grupo de estudio más homogéneo. Se debe mencionar que el control posprocedimiento está protocolizado en la institución, al igual que hay un espacio en la historia clínica dedicado a medidas antropométricas llenado por la mayoría de los médicos, lo que hizo que la cantidad de pacientes excluidos por poca disponibilidad de información fuera baja. A pesar de la existencia de un protocolo estandarizado de bloqueo de ramo medial para la institución, la variabilidad en la respuesta podría depender del operador, teniendo en cuenta el nivel de experiencia o la curva de aprendizaje; este aspecto no puede ser controlado y debe reportarse como una limitante del estudio. Por otro lado, el carácter retrospectivo del estudio hace que se dependa de la calidad de los datos registrados en la historia clínica.

Finalmente, se plantea la necesidad de estudios adicionales de tipo prospectivo, con diseños epidemiológicos que permitan la comparación entre pacientes obesos y no obesos con seguimiento a corto y a largo plazo.

\section{CONCLUSIÓN}

En este estudio encontró que el fallo terapéutico es un fenómeno frecuente posterior al bloqueo de ramo medial lumbar para manejo del dolor crónico axial. No se encontró evidencia de que la obesidad se asocie al fallo terapéutico del bloqueo de ramo medial lumbar bilateral, pero sí se encontró una asociación significativa con el sobrepeso.

\section{RECONOCIMIENTOS}

A las entidades colaboradoras universidad CES, instituto colombiano del dolor INCODOL.

\section{CONFLICTOS DE INTERESES}

Los autores declaran no tener ningún conflicto de intereses.

\section{FINANCIACIÓN}

La presente investigación no ha recibido ayudas específicas provenientes de agencias del sector público, sector comercial o entidades sin ánimo de lucro.

\section{BIBLIOGRAFÍA}

1. Williams A, Wiggers J, O'Brien KM, Wolfenden L, Yoong S, Campbell E, et al. A randomised controlled trial of a lifestyle behavioural intervention for patients with low back pain, who are overweight or obese: study protocol. BMC Musculoskelet Disord. 2016;17(1):70. DOI: 10.1186/s12891-0160922-1.

2. Okifuji $A$, Hare $B$. The association between chronic pain and obesity. J Pain Res. 2015;8:399-408. DOI: 10.2147/JPR. S55598.

3. Manchikanti L, Pampati V, Singh V, Beyer C, Damron K, Fellows B. Evaluation of the role of facet joints in persistent low back pain in obesity: a controlled, prospective, comparative evaluation. Pain Physician. 2001;4(3):266-72.

4. Cohen SP, Huang JHY, Brummett C. Facet joint pain-advances in patient selection and treatment. Nat Rev Rheumatol. 2013;9(2):101-16. DOI: 10.1038/nrrheum.2012.198.

5. Ospina A, Campuzano D, Hincapié E, Vásquez LF, Montoya E, Zapata IC, et al. Eficacia del bloqueo facetario en pacientes con síndrome facetario lumbar. Rev Colomb Anestesiol. 2012;40(3):177-82. DOI: 10.1016/j.rca.2012.06.002.

6. Ellard DR, Underwood M, Achana F, Antrobus JH, Balasubramanian S, Brown S, et al. Facet joint injections for people with persistent non-specific low back pain (Facet Injection Study): a feasibility study for a randomised controlled trial. Health Technol Assess. 2017;21(30):1-184. DOI: 10.3310/hta21300.

7. Manchikanti L, Hirsch JA, Falco FJ, Boswell MV. Management of lumbar zygapophysial (facet) joint pain. World J Orthop. 2016;7(5):315-37. DOI: 10.5312/wjo. v7.i5.315

8. Cohen SP, Doshi TL, Constantinescu OC, Zhao Z, Kurihara C, Larkin TM, et al. Effectiveness of Lumbar Facet Joint Blocks and Predictive Value before Radiofrequency Denervation: The Facet Treatment Study (FACTS), a Randomized, Controlled Clinical Trial. Anesthesiology. 2018;129(3):517-35. DOI: $10.1097 /$ ALN.0000000000002274.

9. Vekaria R, Bhatt R, Ellard DR, Henschke N, Underwood M, Sandhu $\mathrm{H}$. Intra-articular facet joint injections for low back pain: a systematic review. Eur Spine J. 2016;25(4):126681. DOI: 10.1007/s00586-016-4455-y.

10. Santiago AEQ, Leal PC, Bezerra EHM, Giraldes ALA, Ferraro LC, Rezende $A H$, et al. Bloqueo facetario guiado por ultrasonido para lumbalgia: relato de caso. Braz J Anesthesiol. 2014;64(4):278-80. DOI: 10.1016/j.bjane.2012.09.006.

11. Cohen S, Bajwa Z, Kraemer J, Dragovich A, Williams K, Stream J, et al. Factors Predicting Success and Failure for Cervical Facet Radiofrequency Denervation: A Multi-Center Analysis. Reg Anesth Pain Med. 2007;32(6):495-503. DOI: 10.1016/j.rapm.2007.05.009.

12. Ibrahimi-Kacuri D, Murtezani A, Rrecaj S, Martinaj M, Haxhiu B. Low Back Pain and Obesity. Med Arch. 2015;69(2):1146. DOI: 10.5455/medarh.2015.69.114-116.

13. Shiri R, Falah-Hassani K, Heliövaara M, Solovieva S, Amiri S, Lallukka T, et al. Risk Factors for Low Back Pain: A 
Population-Based Longitudinal Study. Arthritis Care Res. 2019;71(2):290-9. DOl: 10.1002/acr.23710.

14. Gomes-Neto M, Lopes JM, Conceição CS, Araujo A, Brasileiro A, Sousa C, et al. Stabilization exercise compared to general exercises or manual therapy for the management of low back pain: A systematic review and meta-analysis. Phys Ther Sport. 2017;23:136-42. DOI: 10.1016/j. ptsp.2016.08.004.

15. da Cruz Fernandes IM, Pinto RZ, Ferreira P, Lira FS. Low back pain, obesity, and inflammatory markers: exercise as potential treatment. J Exerc Rehabil. 2018;14(2):168-74. DOI: 10.12965/jer.1836070.035.
16. Veizi E, Hayek S. Interventional Therapies for Chronic Low Back Pain: Interventional therapies for chronic low back pain. Neuromodulation Technol Neural Interface. 2014;17(Suppl. 2):31-45. DOI: 10.1111/ner.12250.

17. Jorge M. Patarroyo M. Utilidad de los bloqueos anestésicos en patología de columna vertebral en el Hospital Universitario Clínica San Rafael. Rev Fac Med. 2009;17(1):50-4.

18. Martínez-Martínez A, García-Espinosa J, Ruiz-Santiago F, Guzmán-Álvarez L, Castellano-García M. Abordaje intervencionista del síndrome facetario lumbar: Denervación con radiofrecuencia. Rev Chil Radiol. 2017;23(1):7-14. DOI: 10.4067/S0717-93082017000100003. 